\title{
Major Bleeding Events and Associated Factors in a Cohort of Adult Patients Taking Warfarin in an Armed Forces Hospital
}

Gill MS*

Department of Pharmacy, Tuanku Mizan Armed Forces Hospital, Malaysia

*Corresponding author: Manvikram Singh Gill, Department of Pharmacy, Tuanku Mizan Armed Forces Hospital, Ministry of Defence, Kuala Lumpur, Malaysia, Tel: +60103714804; Email: manvikramsinghgill@rocketmail.com

\section{Research Article}

Volume 5 Issue 4

Received Date: November 09, 2020

Published Date: November 18, 2020

DOI: $10.23880 /$ oajds-16000279

\section{Abstract}

Introduction: Warfarin is widely utilized in patients with Atrial Fibrillation (AF) in Malaysia. However, risk of haemorrhage which necessitates monitoring of International Normalised Ratio (INR) and extensive interaction which varies across ethnicity supports the use of Direct Oral Anticoagulants (DOACS). This study is to assess whether demographic data, medical history, and medication history are associated with the risk of major bleeding events.

Methodology: Data was collected retrospectively in a case-controlled environment from the Electronic Medical Record (EMR) database. These patients were attending Medical Out-patient Department (MOPD) clinic, Tuanku Mizan Armed Forces Hospital (TMAFH) from 2nd to 31st January 2018.

Results: Among 60 AF patients reviewed, 83\% had labile INR range and 35\% reported to have 1 or more bleeding event. It is found there is significant association $(\mathrm{p}<0.05)$ for variables of sex, history of stroke, and NSAID usage with the outcome.

Discussion: Majority of patients with major bleeding events are Chinese males. The sample size of the current study is too small to be able to arrive at any conclusive results.

Conclusion: Further studies with bigger sample size are needed among Malaysian Chinese male population. MOPD should establish a warfarin Medication Therapy Adherence Clinic (MTAC) to optimise pharmaceutical care.

Keywords: Major Bleeding Events; Associated Factors; Adult Patients; Atrial Fibrillation, Warfarin

Abbreviations: AF: Atrial Fibrillation; MTAC: Medication Therapy Adherence Clinic; INR: International Normalised Ratio; TMAFH: Tuanku Mizan Armed Forces Hospital; BMI: Body Mass Index; T2DM: Type 2 Diabetes Mellitus; NSAIDs: Non-Steroidal Anti Inflammatory Drugs; TIA: Transient Ischemic Attack; ISTH: International Society on Thrombosis and Haemostasis; EMR: Electronic Medical Record; NYHA: New York Heart Association; DOACS: Direct Oral Anticoagulant Agents.

\section{Introduction}

The risk of stroke and eventually death is increased by Atrial Fibrillation (AF). Vitamin $\mathrm{K}$ antagonists such as the drug warfarin is the standard therapy as it will reduce these risks but may increase the risk of haemorrhage as compared with control treatment [1]. Based on previous studies, warfarin is cumbersome to use because of its multiple interactions with drugs and food [2]. It also requires cyclic laboratory monitoring, increasing the cost of usage indirectly [2]. Hence, it is not often used as rates of therapy incompliance and failure to get the targeted dose within the stipulated time is high [3]. Many patients on warfarin therapy have inadequate anticoagulation as target International Normalised Ratio (INR) varies across ethnicity [3]. Currently in Malaysia, there is a surge of demand to use Direct Oral Anticoagulant Agents (DOACS) that are effective, safe, and convenient to use [4]. However, in Malaysia DOACS 
are still considered a luxury to be prescribed as not many patients can afford its price. Meanwhile Ministry of Health facilities only allocate it for certain group of patients, taking into account the pharmacoeconomics factors. It is important to study what factors in terms of demographic data, medical history and medication history that affect rates of major bleeding events. These factors should be extensively studied to determine which type of population will benefit with recruitment into warfarin Medication Therapy Adherence Clinic (MTAC) or alternatively by considering initiating them with DOACS [5]. Currently, this judgement is made based on HAS-BLED international scoring system [5]. This study is to assess whether demographic data, medical history, and medication history is associated with risk of major bleeding event in patients using warfarin at Tuanku Mizan Armed Forces Hospital (TMAFH).The demographic data such asage, sex and race is considered (Table 1 ).

\begin{tabular}{|c|c|}
\hline Characteristics & p value \\
\hline Sex & $\mathbf{0 . 0 1 9}$ \\
\hline Race & 1.184 \\
\hline Diabetes mellitus & 0.954 \\
\hline Hypertension & 1.484 \\
\hline History of stroke before warfarin & $\mathbf{0 . 0 2 5}$ \\
\hline History of GI bleed & 1.889 \\
\hline Labile INR & 0.132 \\
\hline On aspirin therapy & 0.28 \\
\hline On NSAID therapy & $\mathbf{0 . 0 2 5}$ \\
\hline HAS-BLED risk group & 2.329 \\
\hline Age group & 1.178 \\
\hline
\end{tabular}

Table 1: $p$ value between variables with major bleeding incident(s).

Medical history of patient such as Body Mass Index (BMI) and co morbidities such as history of hypertension and Type 2 Diabetes Mellitus (T2DM) is considered. Medication history such as duration patient taking warfarin with labile INR data is collected. Whether patient is taking concomitant drugs such as aspirin and Non-Steroidal Anti Inflammatory Drugs (NSAIDs) is also noted.

\section{Methodology}

Data was collected retrospectively in a case controlled environment with universal sampling from an Electronic Medical Record (EMR) database of patients attending Medical Out-patient Department (MOPD) clinic in Tuanku Mizan Armed Forces Hospital (TMAFH) over one month of period from 2 nd to 31 st January 2018. In brief, patients were eligible if they had AF documented on electrocardiography performed at clinic screening or within 6 months beforehand and at least one of the following characteristics: previous stroke or Transient Ischemic Attack (TIA), a left ventricular ejection fraction of less than $40 \%$, New York Heart Association (NYHA) Class II or higher heart-failure symptoms within 6 months before screening, and taking the drug warfarin. Reasons for exclusion were the presence of a severe heart-valve disorder, stroke within 14 days or severe stoke within last 6 months. The primary outcome was major bleeding events. Major bleeding event as defined by the International Society on Thrombosis and Haemostasis (ISTH). Statistical analysis was done using SPSS version 23. Chi-square test were used to assess whether demographic data, medical history and medication history is associated with risk of major bleeding events without any stratification or adjustment of other clinical factors for haemorrhage. Statistical significance was set at $\mathrm{p}<0.05$.This study was approved by all appropriate national regulatory authorities and ethics committee of the participating centre. All the authors vouch for the accuracy and completeness of the data and the analyses.

\section{Results}

Among 60 patients reviewed, 35\% reported to have 1 or more bleeding event.

\section{Discussion}

From the EMR database, $60(100 \%)$ patient data was reviewed. These patients are from the range of 40 to 90 years old. Males are 35 (58\%) while females are 25 (42\%). When broken down into race factor, Malays are 23 (38\%), Chinese are $30(50 \%)$ and Indians were 7 (12\%). Majority of participants were Chinese. Substantial incidence of bleeding due to warfarin therapy is a concern among Chinese ethnicity [6]. Studies have suggested that low-intensity anticoagulation is required for this population especially for long term warfarin therapy [7]. This could be due to genetic polymorphism or diet and lifestyle factors [6]. A huge concern is that are the participants also taking Traditional Chinese Medicines (TCM) concurrently with warfarin therapy.

A number of foodstuffs have been implicated in interactions with warfarin. The rate of absorption of dicoumarol can be increased by food. Some of these were not thought to be related to their Vitamin K content. Any big changes in diet have the potential to alter the effects of warfarin on the INR, and it would therefore be prudent to increase monitoring in patients wishing to start a diet. Hence, BMI of patients is a crucial factor to be considered in warfarin therapy patients [8]. The BMI of participants varies.

Co morbidities history of hypertension and T2DM 


\section{Open Access Journal of Dental Sciences}

is a concern. Hypertensive patients are $48(80 \%)$ while T2DM patients are $28(47 \%)$. It is interesting to note that all T2DM patients are also in the hypertensive category. However due to insufficient database in EMR, these patients were not categorised based on blood pressure results or Haemoglobin A1C readings. No baseline values were available. Only $8(13 \%)$ patients had history of stroke before warfarin therapy was initiated. Only 1 patient had history of gastrointestinal (GI) bleeding before warfarin therapy. The estimated glomerular filtration rate (eGFR) of patients also varies. Based on National Kidney Foundation (NKF) Kidney Disease Outcome Quality Initiative (KDOQI) classifications, only $11(18 \%)$ patients had normal results. However, studies have shown that eGFR decreases with age [9]. Due to high number of this study population being elderly, high number are hypertensive patients and insufficient baseline data to compare and contrast from EMR, this data was not thoroughly analysed. However, studies have shown that patients with reduced kidney function needs lower dose of warfarin as they have poor control of anticoagulation and are high risk for hemorrhage [10]. Low-intensity anticoagulation is also required for this population.

The INR data collected shows that $50(83 \%)$ patients had labile INR readings. However, EMR data does not indicate the warfarin dose patient was receiving and INR reading. Local studies have shown that establishment of warfarin MTAC is a good strategy to achieve INR target [5]. MTAC patients managed by physicians, pharmacist and nurses have better INR readings directly achieving pharmaceutical care objectives [5]. Patient taking concomitant drugs that might affect bleeding risk such as aspirin are $23(38 \%)$ while NSAIDs are $8(13 \%)$. However, no data on the dose of aspirin, type of NSAIDs and both drugs frequency and duration patient was exposed is recorded in EMR. These data is crucial to further determine if any drug-drug interaction exist [11]. Warfarin patients should be continuously counseled on the drug-drug interactions that exist with warfarin to increase their knowledge and attitude towards consuming over the counter items [12,13]. From these warfarin patients, 21 (35\%) reported to have encountered a major bleeding event during warfarin therapy. Based on the HAS-BLED score, 1 (2\%) patient risk score $0,10(16 \%)$ patients risk score is 1,21 $(35 \%)$ patients risk score is $2,13(22 \%)$ patients risk score is $3,12(20 \%)$ patients risk score is 4 and $3(5 \%)$ patients risk score is 5 . Number of years patients are on warfarin therapy varies from 5 to 34 years. From the EMR record, 5 (8\%) of patients have died at date of data retrieved. The limitations of this study include small sample size and it is only carried out in one Armed Forces Hospital which is located in Kuala Lumpur. It is proposed that future similar studies with a larger sample size is conducted throughout the armed forces patient population taking warfarin.

\section{Conclusion}

Among $60 \mathrm{AF}$ patients reviewed, 83\% had labile INR range and $35 \%$ reported to have 1 or more bleeding event. This figure is relatively high and this issue needs to be addressed in TMAFH. This figure could be precipitated due to many factors. However, the sample size of the current study is too small to be able to arrive at any conclusive results. Further studies with bigger sample size are needed among TMAFH patients especially among Malaysian Chinese male population. However, this study has identified information that is needed which is incomplete when data is retrospectively retrieved from EMR. This study would recommend for TMAFH to establish a warfarin MTAC. Eventually to do a cross-sectional study comparison between cohorts of patients who choose and don't choose to attend the MTAC.

\section{Acknowledgements}

This work is supported by Commanding Officer of 95 Tuanku Mizan Armed Forces Hospital.

\section{References}

1. Hart RG, Pearce LA, Aguilar MI (2007) Metaanalysis: antithrombotic therapy to prevent stroke in patients who have nonvalvular atrial fibrillation. Ann Intern Med 146(12): 857-867.

2. Hylek EM, Molina EC, Shea C, Henault LE, Regan S (2007) Major hemorrhageand tolerability of warfarin in the first year of therapy among elderly patients with atrial fibrillation. Circulation 115(21): 2689-2696.

3. Ha N, Harun R, Salleh M, Ismail R (2008) The prevalence of CYP2C9 polymorphism in Malaysian, Malay, and Chinese populations. Cardiovascular Revascularization Medicine 9(2): 102.

4. Ezekowitz MD, Reilly PA, Nehmiz, Timothy AS, Rangadham N, et al. (2007) Dabigatran with or without concomitant aspirin compared with warfarin alone in patients with nonvalvular atrialfibrillation (PETRO Study). Am J Cardiol 100(9): 1419-1426.

5. Hasan SS, Shamala R, Syed IA, Basariah N, Chong DWK, et al. (2011) Factors Affecting Warfarin-Related Knowledge and INR Control of Patients Attending Physician- and Pharmacist-Managed Anticoagulation Clinics. J Phar Pract 24(5): 485-493.

6. Chenhsu RY, Chiang SC, Chou MH, Lin MF (2000) LongTerm Treatment with Warfarin in Chinese Population. Ann Pharmacother 34(12): 1395-1401. 


\section{Open Access Journal of Dental Sciences}

7. Thanimalai S, Shafie AA, Hassali MA (2013) Comparing effectiveness of two anticoagulation management models in a Malaysian tertiary hospital. Int J Clin Pharm 35(5): 736-743.

8. Edwards F, Arkell P, Fong AY, Roberts LM, Gendy D, et al. (2013) Bleeding events and associated factors in a cohort of adult patients taking warfarin in Sarawak, Malaysia. J Thromb Thrombolysis 38(2): 226-234.

9. Nita AL, Mark TB, Melissa FB, Joyce AG, Gerald McG, et al. (2009) Kidney Function Influences Warfarin Responsiveness and Hemorrhagic Complications. JASN 20(4): 912-921.

10. Freestone B, Rajaratnam R, Hussain N, Lip GYH (2003) Admissions with atrial fibrillation in a multiracial population in Kuala Lumpur, Malaysia. Int J Cardiol 91(23): 233-238.

11. Connolly SJ, Pogue J, Eikelboom J (2008) Benefit of oral anticoagulant over antiplatelet therapy in atrial fibrillation depends on the quality of international normalized ratio control achieved by centers and countries as measured by time in therapeutic range. Circulation 118: 2029-2037.

12. Hurlen M, Abdelnoor M, Smith P, Erikssen J, Arnesen H (2002) Warfarin, aspirin, or both after myocardial infarction. N Engl J Med 347: 969-974.

13. Birman DE, Radford MJ, Nilasena DS, Gage BF (2006) Use and effectiveness of warfarin in Medicare beneficiaries with atrial fibrillation. Stroke 37(4): 1070-1074. 